\title{
A strong convergence theorem of common elements in Hilbert spaces
}

\section{Zigao Chen}

\section{"Correspondence:}

hschenzg@yeah.net

School of Mathematics and

Information Science, North China

University of Water Resources and

Electric Power, Zhengzhou, Henan

450011, China

\begin{abstract}
The purpose of this article is to investigate the convergence of an iterative process for equilibrium problems, fixed point problems and variational inequalities. Strong convergence of the purposed iterative process is obtained in the framework of Hilbert spaces.
\end{abstract}

MSC: $47 \mathrm{H} 05 ; 47 \mathrm{H} 09 ; 47 \mathrm{~J} 25$

Keywords: equilibrium problem; variational inequality; quasi-nonexpansive; inverse-strongly monotone mapping

\section{Introduction}

Nonlinear analysis plays an important role in optimization problems, economics and transportation. The theory of variational inequalities has emerged as a rapidly growing area of research because of its applications; see [1-17] fore more details and the references therein. To study variational inequalities based on iterative methods has been attracting many authors' attention. For the iterative methods, the most popular method is the Mann iterative method which was introduced by Mann in 1953; see [18] and the references therein. The Mann iterative process has been proved to be weak convergence for nonexpansive mappings in infinite dimension spaces; see [19] and the reference therein. Recently, many authors studied the modification of Mann iterative methods. The most popular one is to use projections. We call the method a hybrid projection method; see [20] and the reference therein. In this paper, we study equilibrium problems, fixed point problems and variational inequalities based on the hybrid projection method. Strong convergence theorems for common solutions of the problems are established in infinite dimension Hilbert spaces.

\section{Preliminaries}

Throughout this paper, we always assume that $H$ is a real Hilbert space with the inner product $\langle\cdot, \cdot\rangle$ and the norm $\|\cdot\|$. Let $C$ be a nonempty closed convex subset of $H$. Let $S: C \rightarrow C$ be a mapping. In this paper, we use $F(S)$ to denote the fixed point set of $S$.

Recall that the mapping $S$ is said to be nonexpansive if

$$
\|S x-S y\| \leq\|x-y\|, \quad \forall x, y \in C .
$$

o 2013 Chen; licensee Springer. This is an Open Access article distributed under the terms of the Creative Commons Attribution License (http://creativecommons.org/licenses/by/2.0), which permits unrestricted use, distribution, and reproduction in any medium, provided the original work is properly cited. 
$S$ is said to be quasi-nonexpansive if $F(S) \neq \emptyset$ and

$$
\|S x-y\| \leq\|x-y\|, \quad \forall x \in C, y \in F(S) .
$$

Let $A: C \rightarrow H$ be a mapping. Recall that $A$ is said to be monotone if

$$
\langle A x-A y, x-y\rangle \geq 0, \quad \forall x, y \in C .
$$

$A$ is said to be strongly monotone if there exists a constant $\alpha>0$ such that

$$
\langle A x-A y, x-y\rangle \geq \alpha\|x-y\|^{2}, \quad \forall x, y \in C .
$$

For such a case, $A$ is also said to be $\alpha$-strongly monotone. $A$ is said to be inverse-strongly monotone if there exists a constant $\alpha>0$ such that

$$
\langle A x-A y, x-y\rangle \geq \alpha\|A x-A y\|^{2}, \quad \forall x, y \in C .
$$

For such a case, $A$ is also said to be $\alpha$-inverse-strongly monotone. $A$ is said to be Lipschitz if there exits a constant $L>0$ such that

$$
\|A x-A y\| \leq L\|x-y\|^{2}, \quad \forall x, y \in C .
$$

For such a case, $A$ is also said to be $L$-Lipschitz. A set-valued mapping $T: H \rightarrow 2^{H}$ is said to be monotone if for all $x, y \in H, f \in T x$ and $g \in T y$ imply $\langle x-y, f-g\rangle>0$. A monotone mapping $T: H \rightarrow 2^{H}$ is maximal if the graph $G(T)$ of $T$ is not properly contained in the graph of any other monotone mapping. It is known that a monotone mapping $T$ is maximal if and only if, for any $(x, f) \in H \times H,\langle x-y, f-g\rangle \geq 0$ for all $(y, g) \in G(T)$ implies $f \in T x$.

Let $F$ be a bifunction of $C \times C$ into $\mathbb{R}$, where $\mathbb{R}$ denotes the set of real numbers and $A$ : $C \rightarrow H$ is an inverse-strongly monotone mapping. In this paper, we consider the following generalized equilibrium problem:

$$
\text { Find } x \in C \text { such that } F(x, y)+\langle A x, y-x\rangle \geq 0, \quad \forall y \in C \text {. }
$$

In this paper, the set of such an $x \in C$ is denoted by $E P(F, A)$, i.e.,

$$
E P(F, A)=\{x \in C: F(x, y)+\langle A x, y-x\rangle \geq 0, \forall y \in C\} .
$$

To study the generalized equilibrium problems (2.1), we may assume that $F$ satisfies the following conditions:

(A1) $F(x, x)=0$ for all $x \in C$;

(A2) $F$ is monotone, i.e., $F(x, y)+F(y, x) \leq 0$ for all $x, y \in C$;

(A3) for each $x, y, z \in C$,

$$
\limsup _{t \downarrow 0} F(t z+(1-t) x, y) \leq F(x, y) ;
$$

(A4) for each $x \in C, y \mapsto F(x, y)$ is convex and lower semi-continuous. 
Next, we give two special cases of the problem (2.1).

(I) If $A \equiv 0$, then the generalized equilibrium problem (2.1) is reduced to the following equilibrium problem:

$$
\text { Find } x \in C \text { such that } F(x, y) \geq 0, \quad \forall y \in C \text {. }
$$

(II) If $F \equiv 0$, then the problem (2.1) is reduced to the following classical variational inequality:

$$
\text { Find } x \in C \text { such that }\langle A x, y-x\rangle \geq 0, \quad \forall y \in C \text {. }
$$

It is known that $x \in C$ is a solution to (2.3) if and only if $x$ is a fixed point of the mapping $P_{C}(I-\lambda A)$, where $\lambda>0$ is a constant and $I$ is the identity mapping.

Recently, many authors studied the problems (2.1), (2.2) and (2.3) based on hybrid projection methods; see, for example, [21-36] and the references therein. Motivated by these results, we investigated the common element problems of the generalized equilibrium problem (2.1) and quasi-nonexpansive mappings based on the shrinking projection algorithm. A strong convergence theorem of common elements is established in the framework of Hilbert spaces.

In order to prove our main results, we also need the following definitions and lemmas.

The following lemma can be found in [4] and [9].

Lemma 2.1 Let $C$ be a nonempty closed convex subset of $H$ and let $F: C \times C \rightarrow \mathbb{R}$ be a bifunction satisfying (A1)-(A4). Then, for any $r>0$ and $x \in H$, there exists $z \in C$ such that

$$
F(z, y)+\frac{1}{r}\langle y-z, z-x\rangle \geq 0, \quad \forall y \in C .
$$

Further, define

$$
T_{r} x=\left\{z \in C: F(z, y)+\frac{1}{r}\langle y-z, z-x\rangle \geq 0, \forall y \in C\right\}
$$

for all $r>0$ and $x \in H$. Then the following hold:

(a) $T_{r}$ is single-valued;

(b) $T_{r}$ is firmly nonexpansive, i.e., for any $x, y \in H$,

$$
\left\|T_{r} x-T_{r} y\right\|^{2} \leq\left\langle T_{r} x-T_{r} y, x-y\right\rangle ;
$$

(c) $F\left(T_{r}\right)=E P(F)$;

(d) $E P(F)$ is closed and convex.

Lemma 2.2 [37] Let B be a monotone mapping of $C$ into $H$ and $N_{C} v$ the normal cone to $C$ at $v \in C$, i.e.,

$$
N_{C} v=\{w \in H:\langle v-u, w\rangle \geq 0, \forall u \in C\}
$$


and define a mapping $M$ on $C$ by

$$
M v= \begin{cases}B v+N_{C} v, & v \in C, \\ \emptyset, & v \notin C .\end{cases}
$$

Then $M$ is maximal monotone and $0 \in M v$ if and only if $\langle B v, u-v\rangle \geq 0$ for all $u \in C$.

\section{Main results}

Theorem 3.1 Let $C$ be a nonempty, closed and convex subset of a real Hilbert space H. Let $F_{m}$ be a bifunction from $C \times C$ to $\mathbb{R}$ which satisfies (A1)-(A4) and $A_{m}: C \rightarrow H$ be a $\kappa_{m}$ inverse-strongly monotone mapping for every $1 \leq m \leq N$, where $N$ denotes some positive integer. Let $S: C \rightarrow C$ be a continuous quasi-nonexpansive mapping which is assumed to be demiclosed at zero and let $B: C \rightarrow H$ be a $\beta$-inverse-strongly monotone mapping. Assume that $\mathcal{F}:=\bigcap_{m=1}^{N} E P\left(F_{m}, A_{m}\right) \cap V I(C, B) \cap F(S) \neq \emptyset$. Let $\left\{\lambda_{n}\right\}$ be a positive sequence in $[0,2 \beta]$ and $\left\{r_{n, m}\right\}$ be a positive sequence in $\left[0,2 \kappa_{m}\right]$ for every $1 \leq m \leq N$. Let $\left\{\alpha_{n}\right\},\left\{\beta_{n, 1}\right\}, \ldots$ and $\left\{\beta_{n, N}\right\}$ be sequences in $[0,1]$. Let $\left\{x_{n}\right\}$ be a sequence generated in the following iterative process:

$$
\left\{\begin{array}{l}
x_{1} \in C \\
C_{1}=C \\
z_{n}=\operatorname{Proj}_{C}\left(\sum_{m=1}^{N} \beta_{n, m} u_{n, m}-\lambda_{n} B \sum_{m=1}^{N} \beta_{n, m} u_{n, m}\right), \\
y_{n}=\alpha_{n} x_{n}+\left(1-\alpha_{n}\right) S z_{n}, \\
C_{n+1}=\left\{v \in C_{n}:\left\|y_{n}-v\right\| \leq\left\|x_{n}-v\right\|\right\} \\
x_{n+1}=P_{C_{n+1}} x_{1}, \quad n \geq 1
\end{array}\right.
$$

where $\left\{u_{n, m}\right\}$ is such that

$$
F_{m}\left(u_{n, m}, u_{m}\right)+\left\langle A_{m} x_{n}, u_{m}-u_{n, m}\right\rangle+\frac{1}{r_{n, m}}\left\langle u_{m}-u_{n, m}, u_{n, m}-x_{n}\right\rangle \geq 0, \quad \forall u_{m} \in C
$$

for each $1 \leq m \leq N$. Assume that the above sequence also satisfies the following restrictions:

(a) $\alpha_{n} \leq a<1$;

(b) $\sum_{m=1}^{N} \beta_{n, m}=1$ and $0 \leq b \leq \beta_{n, m}<1$ for each $1 \leq m \leq N$;

(c) $0<c \leq \lambda_{n} \leq d<2 \beta$ and $0<e \leq r_{n, m} \leq f<2 \kappa_{m}$ for each $1 \leq m \leq N$,

where $a, b, c, d$, e and $f$ are real numbers. Then the sequence $\left\{x_{n}\right\}$ strongly converges to $\operatorname{Proj}_{\mathcal{F}} x_{1}$.

Proof In view of Lemma 2.1, we see that

$$
u_{n, m}=T_{r_{n, m}}\left(x_{n}-r_{n, m} A_{m} x_{n}\right), \quad \forall 1 \leq m \leq N .
$$

Letting $p \in \mathcal{F}$, we obtain that

$$
p=S p=\operatorname{Proj}_{C}\left(I-\lambda_{n} B\right) p=T_{r_{n, m}}\left(p-r_{n, m} A_{m} p\right), \quad \forall m \in\{1,2, \ldots, N\} .
$$


In view of the restriction (c), we obtain that

$$
\begin{aligned}
& \left\|\left(I-r_{n, m} A_{m}\right) x-\left(I-r_{n, m} A_{m}\right) y\right\|^{2} \\
& \quad=\|x-y\|^{2}-2 r_{n, m}\left\langle x-y, A_{m} x-A_{m} y\right\rangle+r_{n, m}^{2}\left\|A_{m} x-A_{m} y\right\|^{2} \\
& \quad \leq\|x-y\|^{2}-r_{n, m}\left(2 \kappa_{m}-r_{n, m}\right)\left\|A_{m} x-A_{m} y\right\|^{2} \\
& \quad \leq\|x-y\|^{2}, \quad \forall x, y \in C .
\end{aligned}
$$

This shows that $I-r_{n, m} A_{m}$ is nonexpansive for every $m \in\{1,2, \ldots, N\}$. In view of the restriction (c), we also see that $I-\lambda_{n} B$ is nonexpansive.

Next, we show that $C_{n}$ is closed and convex. In view of the assumption in the main body of the theorem, we see that $C_{1}=C$ is closed and convex. Suppose that $C_{i}$ is closed and convex for some $i \geq 1$. We show that $C_{i+1}$ is closed and convex for the same $i$. Indeed, for any $v \in C_{i}$, we see that

$$
\left\|y_{i}-v\right\| \leq\left\|x_{i}-v\right\|
$$

is equivalent to

$$
\left\|y_{i}\right\|^{2}-\left\|x_{i}\right\|^{2}-2\left\langle v, y_{i}-x_{i}\right\rangle \geq 0
$$

Thus $C_{i+1}$ is closed and convex. This shows that $C_{n}$ is closed and convex.

Next, we show that $\mathcal{F} \subset C_{n}$ for each $n \geq 1$. From the assumption, we see that $\mathcal{F} \subset C=C_{1}$. Assume that $\mathcal{F} \subset C_{i}$ for some $i \geq 1$. For any $v \in \mathcal{F} \subset C_{i}$, we see that

$$
\begin{aligned}
\left\|y_{i}-v\right\| & =\left\|\alpha_{i} x_{i}+\left(1-\alpha_{i}\right) S z_{i}-v\right\| \\
& \leq \alpha_{i}\left\|x_{i}-v\right\|+\left(1-\alpha_{i}\right)\left\|z_{i}-v\right\| \\
& \leq \alpha_{i}\left\|x_{i}-v\right\|+\left(1-\alpha_{i}\right) \sum_{m=1}^{N} \beta_{i, m}\left\|u_{i, m}-v\right\| \\
& \leq \alpha_{i}\left\|x_{i}-v\right\|+\left(1-\alpha_{i}\right) \sum_{m=1}^{N} \beta_{i, m}\left\|T_{r_{i, m}}\left(I-r_{i, m} A_{m}\right) x_{i}-v\right\| \\
& \leq \alpha_{i}\left\|x_{i}-v\right\|+\left(1-\alpha_{i}\right) \sum_{m=1}^{N} \beta_{i, m}\left\|\left(I-r_{i, m} A_{m}\right) x_{i}-v\right\| \\
& \leq\left\|x_{i}-v\right\| .
\end{aligned}
$$

This shows that $v \in C_{i+1}$. This proves that $\mathcal{F} \subset C_{n}$. Notice that $x_{n}=\operatorname{Proj}_{C_{n}} x_{1}$. For each $v \in \mathcal{F} \subset C_{n}$, we have

$$
\left\|x_{1}-x_{n}\right\| \leq\left\|x_{1}-v\right\|
$$

In particular, we have

$$
\left\|x_{1}-x_{n}\right\| \leq\left\|x_{1}-\operatorname{Proj}_{\mathcal{F}} x_{1}\right\| .
$$


This implies that $\left\{x_{n}\right\}$ is bounded. Since $x_{n}=\operatorname{Proj}_{C_{n}} x_{1}$ and $x_{n+1}=\operatorname{Proj}_{C_{n+1}} x_{1} \in C_{n+1} \subset C_{n}$, we arrive at

$$
0 \leq\left\langle x_{1}-x_{n}, x_{n}-x_{n+1}\right\rangle \leq-\left\|x_{1}-x_{n}\right\|^{2}+\left\|x_{1}-x_{n}\right\|\left\|x_{1}-x_{n+1}\right\| .
$$

It follows that

$$
\left\|x_{n}-x_{1}\right\| \leq\left\|x_{n+1}-x_{1}\right\|
$$

This implies that $\lim _{n \rightarrow \infty}\left\|x_{n}-x_{1}\right\|$ exists. On the other hand, we have

$$
\begin{aligned}
& \left\|x_{n}-x_{n+1}\right\|^{2} \\
& \quad=\left\|x_{n}-x_{1}\right\|^{2}+2\left\langle x_{n}-x_{1}, x_{1}-x_{n+1}\right\rangle+\left\|x_{1}-x_{n+1}\right\|^{2} \\
& \quad=\left\|x_{n}-x_{1}\right\|^{2}-2\left\|x_{n}-x_{1}\right\|^{2}+2\left\langle x_{n}-x_{1}, x_{n}-x_{n+1}\right\rangle+\left\|x_{1}-x_{n+1}\right\|^{2} \\
& \quad \leq\left\|x_{1}-x_{n+1}\right\|^{2}-\left\|x_{n}-x_{1}\right\|^{2} .
\end{aligned}
$$

It follows that

$$
\lim _{n \rightarrow \infty}\left\|x_{n}-x_{n+1}\right\|=0
$$

Notice that $x_{n+1}=\operatorname{Proj}_{C_{n+1}} x_{1} \in C_{n+1}$. It follows that

$$
\left\|y_{n}-x_{n+1}\right\| \leq\left\|x_{n}-x_{n+1}\right\|
$$

This in turn implies that

$$
\left\|y_{n}-x_{n}\right\| \leq\left\|y_{n}-x_{n+1}\right\|+\left\|x_{n}-x_{n+1}\right\| \leq 2\left\|x_{n}-x_{n+1}\right\| .
$$

In view of (3.1), we obtain that

$$
\lim _{n \rightarrow \infty}\left\|x_{n}-y_{n}\right\|=0
$$

On the other hand, we have

$$
\left\|x_{n}-y_{n}\right\|=\left(1-\alpha_{n}\right)\left\|x_{n}-S z_{n}\right\| .
$$

It follows from (3.2) that

$$
\lim _{n \rightarrow \infty}\left\|x_{n}-S z_{n}\right\|=0
$$

For any $p \in \mathcal{F}$, we have from Lemma 2.1 that

$$
\begin{aligned}
\left\|u_{n, m}-p\right\|^{2} & =\left\|T_{r_{n, m}}\left(I-r_{n, m} A_{m}\right) x_{n}-T_{r_{n, m}}\left(I-r_{n, m} A_{m}\right) p\right\|^{2} \\
& \leq\left\|\left(x_{n}-p\right)-r_{n, m}\left(A_{m} x_{n}-A_{m} p\right)\right\|^{2}
\end{aligned}
$$




$$
\begin{aligned}
& =\left\|x_{n}-p\right\|^{2}-2 r_{n, m}\left\langle x_{n}-p, A_{m} x_{n}-A_{m} p\right\rangle+r_{n, m}^{2}\left\|A_{m} x_{n}-A_{m} p\right\|^{2} \\
& \leq\left\|x_{n}-p\right\|^{2}-r_{n, m}\left(2 \kappa_{m}-r_{n, m}\right)\left\|A_{m} x_{n}-A_{m} p\right\|^{2}, \\
& \quad \forall m \in\{1,2, \ldots, N\} .
\end{aligned}
$$

On the other hand, we have

$$
\begin{aligned}
\left\|y_{n}-p\right\|^{2} & =\left\|\alpha_{n} x_{n}+\left(1-\alpha_{n}\right) S z_{n}-p\right\|^{2} \\
& \leq \alpha_{n}\left\|x_{n}-p\right\|^{2}+\left(1-\alpha_{n}\right)\left\|S z_{n}-p\right\|^{2} \\
& \leq \alpha_{n}\left\|x_{n}-p\right\|^{2}+\left(1-\alpha_{n}\right)\left\|z_{n}-p\right\|^{2} \\
& \leq \alpha_{n}\left\|x_{n}-p\right\|^{2}+\left(1-\alpha_{n}\right) \sum_{m=1}^{N} \beta_{n, m}\left\|u_{n, m}-p\right\|^{2} .
\end{aligned}
$$

Substituting (3.4) into (3.5), we arrive at

$$
\left\|y_{n}-p\right\|^{2} \leq\left\|x_{n}-p\right\|^{2}-\left(1-\alpha_{n}\right) \sum_{m=1}^{N} \beta_{n, m} r_{n, m}\left(2 \kappa_{m}-r_{n, m}\right)\left\|A_{m} x_{n}-A_{m} p\right\|^{2}
$$

This in turn implies that

$$
\begin{aligned}
& \left(1-\alpha_{n}\right) \beta_{n, m} r_{n, m}\left(2 \kappa_{m}-r_{n, m}\right)\left\|A_{m} x_{n}-A_{m} p\right\|^{2} \\
& \quad \leq\left\|x_{n}-p\right\|^{2}-\left\|y_{n}-p\right\|^{2} \\
& \quad \leq\left(\left\|x_{n}-p\right\|+\left\|y_{n}-p\right\|\right)\left\|x_{n}-y_{n}\right\|, \quad \forall m \in\{1,2, \ldots, N\} .
\end{aligned}
$$

In view of the restrictions (a)-(c), we obtain from (3.2) that

$$
\lim _{n \rightarrow \infty}\left\|A_{m} x_{n}-A_{m} p\right\|=0, \quad \forall m \in\{1,2, \ldots, N\}
$$

On the other hand, we have from Lemma 2.1 that

$$
\begin{aligned}
\left\|u_{n, m}-p\right\|^{2}= & \left\|T_{r_{n, m}}\left(I-r_{n, m} A_{m}\right) x_{n}-T_{r_{n, m}}\left(I-r_{n, m} A_{m}\right) p\right\|^{2} \\
\leq & \left\langle\left(I-r_{n, m} A_{m}\right) x_{n}-\left(I-r_{n, m} A_{m}\right) p, u_{n, m}-p\right\rangle \\
= & \frac{1}{2}\left(\left\|\left(I-r_{n, m} A_{m}\right) x_{n}-\left(I-r_{n, m} A_{m}\right) p\right\|^{2}+\left\|u_{n, m}-p\right\|^{2}\right. \\
& \left.-\left\|\left(I-r_{n, m} A_{m}\right) x_{n}-\left(I-r_{n, m} A_{m}\right) p-\left(u_{n, m}-p\right)\right\|^{2}\right) \\
\leq & \frac{1}{2}\left(\left\|x_{n}-p\right\|^{2}+\left\|u_{n, m}-p\right\|^{2}-\left\|x_{n}-u_{n, m}-r_{n, m}\left(A_{m} x_{n}-A_{m} p\right)\right\|^{2}\right) \\
= & \frac{1}{2}\left(\left\|x_{n}-p\right\|^{2}+\left\|u_{n, m}-p\right\|^{2}-\left(\left\|x_{n}-u_{n, m}\right\|^{2}\right.\right. \\
& \left.\left.-2 r_{n, m}\left\langle x_{n}-u_{n, m}, A_{m} x_{n}-A_{m} p\right\rangle+r_{n, m}^{2}\left\|A_{m} x_{n}-A_{m} p\right\|^{2}\right)\right) .
\end{aligned}
$$


This implies that

$$
\left\|u_{n, m}-p\right\|^{2} \leq\left\|x_{n}-p\right\|^{2}-\left\|x_{n}-u_{n, m}\right\|^{2}+2 r_{n, m}\left\|x_{n}-u_{n, m}\right\|\left\|A_{m} x_{n}-A_{m} p\right\| .
$$

Notice that

$$
\begin{aligned}
\left\|y_{n}-p\right\|^{2} & \leq \alpha_{n}\left\|x_{n}-p\right\|^{2}+\left(1-\alpha_{n}\right)\left\|S z_{n}-p\right\|^{2} \\
& \leq \alpha_{n}\left\|x_{n}-p\right\|^{2}+\left(1-\alpha_{n}\right)\left\|z_{n}-p\right\|^{2} \\
& \leq \alpha_{n}\left\|x_{n}-p\right\|^{2}+\left(1-\alpha_{n}\right) \sum_{m=1}^{N} \beta_{n, m}\left\|u_{n, m}-p\right\|^{2} .
\end{aligned}
$$

Substituting (3.8) into (3.9), we see that

$$
\begin{aligned}
\left\|y_{n}-p\right\|^{2} \leq & \left\|x_{n}-p\right\|^{2}+\left(1-\alpha_{n}\right) \sum_{m=1}^{N} \beta_{n, m} 2 r_{n, m}\left\|x_{n}-u_{n, m}\right\|\left\|A_{m} x_{n}-A_{m} p\right\| \\
& -\left(1-\alpha_{n}\right) \sum_{m=1}^{N} \beta_{n, m}\left\|x_{n}-u_{n, m}\right\|^{2} \\
\leq & \left\|x_{n}-p\right\|^{2}+\sum_{m=1}^{N} 2 r_{n, m}\left\|x_{n}-u_{n, m}\right\|\left\|A_{m} x_{n}-A_{m} p\right\| \\
& \quad-\left(1-\alpha_{n}\right) \sum_{m=1}^{N} \beta_{n, m}\left\|x_{n}-u_{n, m}\right\|^{2}, \quad \forall 1 \leq m \leq N .
\end{aligned}
$$

It follows that

$$
\begin{aligned}
& \left(1-\alpha_{n}\right) \beta_{n, m}\left\|x_{n}-u_{n, m}\right\|^{2} \\
& \leq\left\|x_{n}-p\right\|^{2}-\left\|y_{n}-p\right\|^{2}+\sum_{m=1}^{N} 2 r_{n, m}\left\|x_{n}-u_{n, m}\right\|\left\|A_{m} x_{n}-A_{m} p\right\| \\
& \leq\left(\left\|x_{n}-p\right\|+\left\|y_{n}-p\right\|\right)\left\|x_{n}-y_{n}\right\|+\sum_{m=1}^{N} 2 r_{n, m}\left\|x_{n}-u_{n, m}\right\|\left\|A_{m} x_{n}-A_{m} p\right\|, \\
& \quad \forall 1 \leq m \leq N .
\end{aligned}
$$

In view of the restrictions (a) and (b), we obtain from (3.2) and (3.7) that

$$
\lim _{n \rightarrow \infty}\left\|x_{n}-u_{n, m}\right\|=0, \quad \forall 1 \leq m \leq N
$$

Since $\left\{x_{n}\right\}$ is bounded, we may assume that there is a subsequence $\left\{x_{n_{i}}\right\}$ of $\left\{x_{n}\right\}$ converging weakly to some point $x$. It follows from (3.12) that $u_{n_{i}, m}$ converges weakly to $x$ for every $m \in\{1,2, \ldots, N\}$.

Next, we show that $x \in E P\left(F_{m}, A_{m}\right)$ for every $m \in\{1,2, \ldots, N\}$. Since $u_{n, m}=T_{r_{n, m}}\left(x_{n}-\right.$ $\left.r_{n, m} A_{m} x_{n}\right)$ for any $u \in C$, we have

$$
F_{m}\left(u_{n, m}, u_{m}\right)+\left\langle A_{m} x_{n}, u_{m}-u_{n, m}\right\rangle+\frac{1}{r_{n, m}}\left\langle u_{m}-u_{n, m}, u_{n, m}-x_{n}\right\rangle \geq 0 .
$$


From the condition (A2), we see that

$$
\left\langle A_{m} x_{n}, u_{m}-u_{n, m}\right\rangle+\frac{1}{r_{n, m}}\left\langle u_{m}-u_{n, m}, u_{n, m}-x_{n}\right\rangle \geq F_{m}\left(u_{m}, u_{n, m}\right) .
$$

Replacing $n$ by $n_{i}$, we arrive at

$$
\left\langle A_{m} x_{n_{i}}, u_{m}-u_{n_{i}, m}\right\rangle+\left\langle u_{m}-u_{n_{i}, m}, \frac{u_{n_{i}, m}-x_{n_{i}}}{r_{n_{i}, m}}\right\rangle \geq F_{m}\left(u_{m}, u_{n_{i}, m}\right)
$$

For $t_{m}$ with $0<t_{m} \leq 1$ and $u_{m} \in C$, let $u_{t_{m}}=t_{m} u_{m}+\left(1-t_{m}\right) x$. Since $u_{m} \in C$ and $x \in C$, we have $u_{t_{m}} \in C$ for every $1 \leq m \leq N$. It follows from (3.14) that

$$
\begin{aligned}
\left\langle u_{t_{m}}\right. & \left.-u_{n_{i}, m}, A_{m} u_{t_{m}}\right\rangle \\
\geq & \left\langle u_{t_{m}}-u_{n_{i}, m}, A_{m} u_{t_{m}}\right\rangle-\left\langle A_{m} x_{n_{i}, m}, u_{t_{m}}-u_{n_{i}, m}\right\rangle-\left\langle u_{t_{m}}-u_{n_{i}, m}, \frac{u_{n_{i}, m}-x_{n_{i}}}{r_{n_{i}, m}}\right\rangle \\
& +F_{m}\left(u_{t_{m}}, u_{n_{i}, m}\right) \\
= & \left\langle u_{t_{m}}-u_{n_{i}, m}, A_{m} u_{t_{m}}-A_{m} u_{n_{i}, m}\right\rangle+\left\langle u_{t_{m}}-u_{n_{i}, m}, A_{m} u_{n_{i}, m}-A_{m} x_{n_{i}}\right\rangle \\
& -\left\langle u_{t_{m}}-u_{n_{i}, m}, \frac{u_{n_{i}, m}-x_{n_{i}}}{r_{n_{i}, m}}\right\rangle+F_{m}\left(u_{t_{m}}, u_{n_{i}, m}\right) .
\end{aligned}
$$

From (3.12), we have $A_{m} u_{n_{i}, m}-A_{m} x_{n_{i}} \rightarrow 0$ as $i \rightarrow \infty$ for every $1 \leq m \leq N$. On the other hand, we obtain from the monotonicity of $A_{m}$ that $\left\langle u_{t_{m}}-u_{n_{i}, m}, A_{m} u_{t_{m}}-A_{m} u_{n_{i}, m}\right\rangle \geq 0$. It follows from (A4) that

$$
\left\langle u_{t_{m}}-x, A_{m} u_{t_{m}}\right\rangle \geq F_{m}\left(u_{t_{m}}, x\right), \quad \forall 1 \leq m \leq N
$$

From (A1) and (A4), we obtain from (3.16) that

$$
\begin{aligned}
0 & =F_{m}\left(u_{t_{m}}, u_{t_{m}}\right) \leq t_{m} F_{m}\left(u_{t_{m}}, u_{m}\right)+\left(1-t_{m}\right) F_{m}\left(u_{t_{m}}, x\right) \\
& \leq t_{m} F_{m}\left(u_{t_{m}}, u_{m}\right)+\left(1-t_{m}\right)\left\langle u_{t_{m}}-x, A_{m} u_{t_{m}}\right\rangle \\
& =t_{m} F_{m}\left(u_{t_{m}}, u_{m}\right)+\left(1-t_{m}\right) t_{m}\left\langle u_{m}-x, A_{m} u_{t_{m}}\right\rangle,
\end{aligned}
$$

which yields that

$$
F_{m}\left(u_{t_{m}}, u_{m}\right)+\left(1-t_{m}\right)\left\langle u_{m}-x, A_{m} u_{t_{m}}\right\rangle \geq 0, \quad \forall 1 \leq m \leq N
$$

Letting $t_{m} \rightarrow 0$ in the above inequality for every $1 \leq m \leq N$, we arrive at

$$
F_{m}\left(x, u_{m}\right)+\left\langle u_{m}-x, A_{m} \xi\right\rangle \geq 0, \quad \forall 1 \leq m \leq N
$$

This shows that $x \in E P\left(F_{m}, A_{m}\right)$ for every $1 \leq m \leq N$, that is, $x \in \bigcap_{m=1}^{N} E P\left(F_{m}, A_{m}\right)$. Putting $w_{n}=\sum_{m=1}^{N} \beta_{n, m} u_{n, m}$, we see that

$$
\left\|w_{n}-p\right\| \leq\left\|x_{n}-p\right\|
$$


and

$$
\begin{aligned}
\left\|y_{n}-p\right\|^{2} & =\left\|\alpha_{n} x_{n}+\left(1-\alpha_{n}\right) S z_{n}-p\right\|^{2} \\
& \leq \alpha_{n}\left\|x_{n}-p\right\|^{2}+\left(1-\alpha_{n}\right)\left\|S z_{n}-p\right\|^{2} \\
& \leq \alpha_{n}\left\|x_{n}-p\right\|^{2}+\left(1-\alpha_{n}\right)\left\|z_{n}-p\right\|^{2} \\
& \leq \alpha_{n}\left\|x_{n}-p\right\|^{2}+\left(1-\alpha_{n}\right)\left\|\left(I-\lambda_{n} B\right) w_{n}-p\right\|^{2} \\
& \leq\left\|x_{n}-p\right\|^{2}-\left(1-\alpha_{n}\right) \lambda_{n}\left(2 \beta-\lambda_{n}\right)\left\|B w_{n}-B p\right\|^{2} .
\end{aligned}
$$

This in turn implies that

$$
\begin{aligned}
\left(1-\alpha_{n}\right) \lambda_{n}\left(2 \beta-\lambda_{n}\right)\left\|B w_{n}-B p\right\|^{2} & \leq\left\|x_{n}-p\right\|^{2}-\left\|y_{n}-p\right\|^{2} \\
& \leq\left(\left\|x_{n}-p\right\|+\left\|y_{n}-p\right\|\right)\left\|x_{n}-y_{n}\right\| .
\end{aligned}
$$

In view of the restriction (a)-(c), we obtain from (3.2) that

$$
\lim _{n \rightarrow \infty}\left\|B w_{n}-B p\right\|=0
$$

On the other hand, we have from the firm nonexpansivity of $\operatorname{Proj}_{C}$ that

$$
\begin{aligned}
\left\|z_{n}-p\right\|^{2}= & \left\|\operatorname{Proj}_{C}\left(I-\lambda_{n} B\right) w_{n}-\operatorname{Proj}_{C}\left(I-\lambda_{n} B\right) p\right\|^{2} \\
\leq & \left\langle\left(I-\lambda_{n} B\right) w_{n}-\left(I-\lambda_{n} B\right) p, z_{n}-p\right\rangle \\
= & \frac{1}{2}\left(\left\|\left(I-\lambda_{n} B\right) w_{n}-\left(I-\lambda_{n} B\right) p\right\|^{2}+\left\|z_{n}-p\right\|^{2}\right. \\
& \left.-\left\|\left(I-\lambda_{n} B\right) w_{n}-\left(I-\lambda_{n} B\right) p-\left(z_{n}-p\right)\right\|^{2}\right) \\
\leq & \frac{1}{2}\left(\left\|w_{n}-p\right\|^{2}+\left\|z_{n}-p\right\|^{2}-\left\|w_{n}-z_{n}-\lambda_{n}\left(B w_{n}-B p\right)\right\|^{2}\right) \\
\leq & \frac{1}{2}\left(\left\|x_{n}-p\right\|^{2}+\left\|z_{n}-p\right\|^{2}-\left\|w_{n}-z_{n}\right\|^{2}\right. \\
& \left.+2 \lambda_{n}\left\langle w_{n}-z_{n}, B w_{n}-B p\right\rangle-\lambda_{n}^{2}\left\|B w_{n}-B p\right\|^{2}\right) .
\end{aligned}
$$

This implies that

$$
\left\|z_{n}-p\right\|^{2} \leq\left\|x_{n}-p\right\|^{2}-\left\|w_{n}-z_{n}\right\|^{2}+2 \lambda_{n}\left\|w_{n}-z_{n}\right\|\left\|B w_{n}-B p\right\|,
$$

from which it follows that

$$
\begin{aligned}
\left\|y_{n}-p\right\|^{2} \leq & \alpha_{n}\left\|x_{n}-p\right\|^{2}+\left(1-\alpha_{n}\right)\left\|S z_{n}-p\right\|^{2} \\
\leq & \alpha_{n}\left\|x_{n}-p\right\|^{2}+\left(1-\alpha_{n}\right)\left\|z_{n}-p\right\|^{2} \\
\leq & \left\|x_{n}-p\right\|^{2}-\left(1-\alpha_{n}\right)\left\|w_{n}-z_{n}\right\|^{2} \\
& +2 \lambda_{n}\left\|w_{n}-z_{n}\right\|\left\|B w_{n}-B p\right\| .
\end{aligned}
$$


Hence, we get that

$$
\begin{aligned}
& \left(1-\alpha_{n}\right)\left\|w_{n}-z_{n}\right\|^{2} \\
& \quad \leq\left\|x_{n}-p\right\|^{2}-\left\|y_{n}-p\right\|^{2}+2 \lambda_{n}\left\|w_{n}-z_{n}\right\|\left\|B w_{n}-B p\right\| \\
& \quad \leq\left(\left\|x_{n}-p\right\|+\left\|y_{n}-p\right\|\right)\left\|x_{n}-y_{n}\right\|+2 \lambda_{n}\left\|w_{n}-z_{n}\right\|\left\|B w_{n}-B p\right\| .
\end{aligned}
$$

In view of the restriction (a), we obtain from (3.2) and (3.19) that

$$
\lim _{n \rightarrow \infty}\left\|w_{n}-z_{n}\right\|=0
$$

Note that

$$
\left\|z_{n}-x_{n}\right\| \leq\left\|z_{n}-w_{n}\right\|+\left\|w_{n}-x_{n}\right\| \leq\left\|z_{n}-w_{n}\right\|+\sum_{m=1}^{N} \beta_{n, m}\left\|u_{n, m}-x_{n}\right\|
$$

In view of (3.12) and (3.20), we get that

$$
\lim _{n \rightarrow \infty}\left\|z_{n}-x_{n}\right\|=0
$$

Next, we prove that $x \in V I(C, B)$. In fact, let $M$ be the maximal monotone mapping defined by

$$
M y= \begin{cases}B y+N_{C} y, & y \in C, \\ \emptyset, & y \notin C .\end{cases}
$$

For any given $(s, t) \in G(T)$, we have $t-B s \in N_{C} s$. Since $z_{n} \in C$, by the definition of $N_{C}$, we have

$$
\left\langle s-z_{n}, t-B s\right\rangle \geq 0
$$

In view of the algorithm, we obtain that

$$
\left\langle s-z_{n}, z_{n}-\left(I-\lambda_{n} B\right) w_{n}\right\rangle \geq 0
$$

and hence

$$
\left\langle s-z_{n}, \frac{z_{n}-w_{n}}{\lambda_{n}}+B w_{n}\right\rangle \geq 0
$$

Since $B$ is monotone, we obtain from (3.23) that

$$
\begin{aligned}
\left\langle s-z_{n_{i}}, t\right\rangle & \geq\left\langle s-z_{n_{i}}, B s\right\rangle \\
& \geq\left\langle s-z_{n_{i}}, B s\right\rangle-\left\langle s-z_{n_{i}}, \frac{z_{n_{i}}-w_{n_{i}}}{\lambda_{n_{i}}}+B w_{n_{i}}\right\rangle \\
& =\left\langle s-z_{n_{i}}, B s-B z_{n_{i}}\right\rangle+\left\langle s-z_{n_{i}}, B z_{n_{i}}-B w_{n_{i}}\right\rangle
\end{aligned}
$$




$$
\begin{aligned}
& -\left\langle s-z_{n_{i}}, \frac{z_{n_{i}}-w_{n_{i}}}{\lambda_{n_{i}}}\right\rangle \\
\geq & \left\langle s-z_{n_{i}}, B z_{n_{i}}-B w_{n_{i}}\right\rangle-\left\langle s-z_{n_{i}}, \frac{z_{n_{i}}-w_{n_{i}}}{\lambda_{n_{i}}}\right\rangle .
\end{aligned}
$$

It follows from (3.21) that $z_{n_{i}} \rightarrow x$. On the other hand, we have that $B$ is $\frac{1}{\beta}$-Lipschitz continuous. It follows from (3.20) that

$$
\langle s-x, t\rangle \geq 0
$$

Notice that $M$ is maximal monotone and hence $0 \in M x$. This shows that $x \in V I(C, B)$. Notice that

$$
\left\|x_{n}-S x_{n}\right\| \leq\left\|x_{n}-S z_{n}\right\|+\left\|S z_{n}-S x_{n}\right\|
$$

We find from (3.3) and (3.21) that

$$
\lim _{n \rightarrow \infty}\left\|x_{n}-S x_{n}\right\|=0
$$

Next, we prove that $x \in F(S)$. Since $S$ is demiclosed at zero, we see that $x \in F(S)$. This proves that $x \in \mathcal{F}$. Notice that $\operatorname{Proj}_{\mathcal{F}} x_{1} \subset C_{n+1}$ and $x_{n+1}=\operatorname{Proj}_{C_{n+1}} x_{1}$, we have

$$
\left\|x_{1}-x_{n+1}\right\| \leq\left\|x_{1}-\operatorname{Proj}_{\mathcal{F}} x_{1}\right\|
$$

On the other hand, we have

$$
\begin{aligned}
\left\|x_{1}-\operatorname{Proj}_{\mathcal{F}} x_{1}\right\| & \leq\left\|x_{1}-x\right\| \\
& \leq \liminf _{i \rightarrow \infty}\left\|x_{1}-x_{n_{i}}\right\| \\
& \leq \limsup _{i \rightarrow \infty}\left\|x_{1}-x_{n_{i}}\right\| \\
& \leq\left\|x_{1}-\operatorname{Proj}_{\mathcal{F}} x_{1}\right\| .
\end{aligned}
$$

We, therefore, obtain that

$$
\left\|x_{1}-x\right\|=\lim _{i \rightarrow \infty}\left\|x_{1}-x_{n_{i}}\right\|=\left\|x_{1}-\operatorname{Proj}_{\mathcal{F}} x_{1}\right\|
$$

This implies $x_{n_{i}} \rightarrow x=\operatorname{Proj}_{\mathcal{F}} x_{1}$. Since $\left\{x_{n_{i}}\right\}$ is an arbitrary subsequence of $\left\{x_{n}\right\}$, we obtain that $x_{n} \rightarrow \operatorname{Proj}_{\mathcal{F}} x_{1}$ as $n \rightarrow \infty$. This completes the proof.

If $S$ is an identity mapping, we obtain from Theorem 3.1 the following.

Corollary 3.2 Let $C$ be a nonempty, closed and convex subset of a real Hilbert space $H$. Let $F_{m}$ be a bifunction from $C \times C$ to $\mathbb{R}$ which satisfies (A1)-(A4) and $A_{m}: C \rightarrow H$ be a $\kappa_{m}$-inverse-strongly monotone mapping for every $1 \leq m \leq N$, where $N$ denotes some positive integer. Let $B: C \rightarrow H$ be a $\beta$-inverse-strongly monotone mapping. Assume that $\mathcal{F}:=\bigcap_{m=1}^{N} E P\left(F_{m}, A_{m}\right) \cap V I(C, B) \neq \emptyset$. Let $\left\{\lambda_{n}\right\}$ be a positive sequence in $[0,2 \beta]$ and $\left\{r_{n, m}\right\}$ 
be a positive sequence in $\left[0,2 \kappa_{m}\right]$ for every $1 \leq m \leq N$. Let $\left\{\alpha_{n}\right\},\left\{\beta_{n, 1}\right\}, \ldots$ and $\left\{\beta_{n, N}\right\}$ be sequences in $[0,1]$. Let $\left\{x_{n}\right\}$ be a sequence generated in the following iterative process:

$$
\left\{\begin{array}{l}
x_{1} \in C, \\
C_{1}=C, \\
y_{n}=\alpha_{n} x_{n}+\left(1-\alpha_{n}\right) \operatorname{Proj}_{C}\left(\sum_{m=1}^{N} \beta_{n, m} u_{n, m}-\lambda_{n} B \sum_{m=1}^{N} \beta_{n, m} u_{n, m}\right), \\
C_{n+1}=\left\{v \in C_{n}:\left\|y_{n}-v\right\| \leq\left\|x_{n}-v\right\|\right\}, \\
x_{n+1}=P_{C_{n+1}} x_{1}, \quad n \geq 1,
\end{array}\right.
$$

where $\left\{u_{n, m}\right\}$ is such that

$$
F_{m}\left(u_{n, m}, u_{m}\right)+\left\langle A_{m} x_{n}, u_{m}-u_{n, m}\right\rangle+\frac{1}{r_{n, m}}\left\langle u_{m}-u_{n, m}, u_{n, m}-x_{n}\right\rangle \geq 0, \quad \forall u_{m} \in C
$$

for each $1 \leq m \leq N$. Assume that the above sequence also satisfies the following restrictions:

(a) $\alpha_{n} \leq a<1$;

(b) $\sum_{m=1}^{N} \beta_{n, m}=1$ and $0 \leq b \leq \beta_{n, m}<1$ for each $1 \leq m \leq N$;

(c) $0<c \leq \lambda_{n} \leq d<2 \beta$ and $0<e \leq r_{n, m} \leq f<2 \kappa_{m}$ for each $1 \leq m \leq N$,

where $a, b, c, d$, e and $f$ are real numbers. Then the sequence $\left\{x_{n}\right\}$ strongly converges to $\operatorname{Proj}_{\mathcal{F}} x_{1}$.

If $N=1$, we obtain from Theorem 3.1 the following.

Corollary 3.3 Let $C$ be a nonempty, closed and convex subset of a real Hilbert space $H$. Let $F$ be a bifunction from $C \times C$ to $\mathbb{R}$ which satisfies (A1)-(A4) and $A: C \rightarrow H$ be a $\kappa$ inverse-strongly monotone mapping. Let $S: C \rightarrow C$ be a continuous quasi-nonexpansive mapping which is assumed to be demiclosed at zero and let $B: C \rightarrow H$ be a $\beta$-inversestrongly monotone mapping. Assume that $\mathcal{F}:=E P(F, A) \cap V I(C, B) \cap F(S) \neq \emptyset$. Let $\left\{\lambda_{n}\right\}$ be a positive sequence in $[0,2 \beta]$ and $\left\{r_{n}\right\}$ be a positive sequence in $[0,2 \kappa]$. Let $\left\{\alpha_{n}\right\}$ be a sequence in $[0,1]$. Let $\left\{x_{n}\right\}$ be a sequence generated in the following iterative process:

$$
\left\{\begin{array}{l}
x_{1} \in C \\
C_{1}=C \\
y_{n}=\alpha_{n} x_{n}+\left(1-\alpha_{n}\right) S \operatorname{Proj}_{C}\left(u_{n}-\lambda_{n} B u_{n}\right) \\
C_{n+1}=\left\{v \in C_{n}:\left\|y_{n}-v\right\| \leq\left\|x_{n}-v\right\|\right\} \\
x_{n+1}=P_{C_{n+1}} x_{1}, \quad n \geq 1
\end{array}\right.
$$

where $\left\{u_{n}\right\}$ is such that

$$
F\left(u_{n}, u\right)+\left\langle A x_{n}, u-u_{n}\right\rangle+\frac{1}{r_{n}}\left\langle u-u_{n}, u_{n}-x_{n}\right\rangle \geq 0, \quad \forall u_{m} \in C .
$$

Assume that the above sequence also satisfies the following restrictions:

(a) $\alpha_{n} \leq a<1$;

(b) $0<b \leq \lambda_{n} \leq c<2 \beta$ and $0<d \leq r_{n} \leq e<2 \kappa$ for each $1 \leq m \leq N$, 
where $a, b, c, d$ and e are real numbers. Then the sequence $\left\{x_{n}\right\}$ strongly converges to $\operatorname{Proj}_{\mathcal{F}} x_{1}$.

If $B$ is a zero operator, we obtain from Theorem 3.1 the following.

Corollary 3.4 Let $C$ be a nonempty, closed and convex subset of a real Hilbert space $H$. Let $F_{m}$ be a bifunction from $C \times C$ to $\mathbb{R}$ which satisfies (A1)-(A4) and $A_{m}: C \rightarrow H$ be a $\kappa_{m}$ inverse-strongly monotone mapping for every $1 \leq m \leq N$, where $N$ denotes some positive integer. Let $S: C \rightarrow C$ be a continuous quasi-nonexpansive mapping which is assumed to be demiclosed at zero. Assume that $\mathcal{F}:=\bigcap_{m=1}^{N} E P\left(F_{m}, A_{m}\right) \cap F(S) \neq \emptyset$. Let $\left\{r_{n, m}\right\}$ be a positive sequence in $\left[0,2 \kappa_{m}\right]$ for every $1 \leq m \leq N$. Let $\left\{\alpha_{n}\right\},\left\{\beta_{n, 1}\right\}, \ldots$ and $\left\{\beta_{n, N}\right\}$ be sequences in $[0,1]$. Let $\left\{x_{n}\right\}$ be a sequence generated in the following iterative process:

$$
\left\{\begin{array}{l}
x_{1} \in C, \\
C_{1}=C, \\
y_{n}=\alpha_{n} x_{n}+\left(1-\alpha_{n}\right) S \sum_{m=1}^{N} \beta_{n, m} u_{n, m}, \\
C_{n+1}=\left\{v \in C_{n}:\left\|y_{n}-v\right\| \leq\left\|x_{n}-v\right\|\right\}, \\
x_{n+1}=P_{C_{n+1}} x_{1}, \quad n \geq 1,
\end{array}\right.
$$

where $\left\{u_{n, m}\right\}$ is such that

$$
F_{m}\left(u_{n, m}, u_{m}\right)+\left\langle A_{m} x_{n}, u_{m}-u_{n, m}\right\rangle+\frac{1}{r_{n, m}}\left\langle u_{m}-u_{n, m}, u_{n, m}-x_{n}\right\rangle \geq 0, \quad \forall u_{m} \in C
$$

for each $1 \leq m \leq N$. Assume that the above sequence also satisfies the following restrictions:

(a) $\alpha_{n} \leq a<1$;

(b) $\sum_{m=1}^{N} \beta_{n, m}=1$ and $0 \leq b \leq \beta_{n, m}<1$ for each $1 \leq m \leq N$;

(c) $0<c \leq r_{n, m} \leq d<2 \kappa_{m}$ for each $1 \leq m \leq N$,

where $a, b, c$ and $d$ are real numbers. Then the sequence $\left\{x_{n}\right\}$ strongly converges to $\operatorname{Proj}_{\mathcal{F}} x_{1}$.

Finally, we consider the following optimization problem: Find an $x^{*}$ such that

$$
\left\{\begin{array}{l}
\varphi_{1}\left(x^{*}\right)=\min _{x \in C} \varphi_{1}(x), \\
\varphi_{2}\left(x^{*}\right)=\min _{x \in C} \varphi_{2}(x), \\
\vdots \\
\varphi_{N}\left(x^{*}\right)=\min _{x \in C} \varphi_{N}(x),
\end{array}\right.
$$

where $\varphi_{m}: C \rightarrow \mathbb{R}$ is a convex and lower semicontinuous function for each $1 \leq m \leq N$, where $N \geq 1$ is some positive integer.

Theorem 3.5 Let $C$ be a nonempty, closed and convex subset of a real Hilbert space $H$. Let $\varphi_{m}$ be a proper convex and lower semicontinuous function for every $1 \leq m \leq N$, where $N$ denotes some positive integer. Assume that $\mathcal{F}:=O P(\varphi) \cap V I(C, B) \cap F(S) \neq \emptyset, O P(\varphi)$ denotes the solution set of the above optimization problem. Let $\left\{\alpha_{n}\right\},\left\{\beta_{n, 1}\right\}, \ldots$ and $\left\{\beta_{n, N}\right\}$ be 
sequences in $[0,1]$. Let $\left\{x_{n}\right\}$ be a sequence generated in the following iterative process:

$$
\left\{\begin{array}{l}
x_{1} \in C, \\
C_{1}=C, \\
y_{n}=\alpha_{n} x_{n}+\left(1-\alpha_{n}\right) \sum_{m=1}^{N} \beta_{n, m} u_{n, m}, \\
C_{n+1}=\left\{v \in C_{n}:\left\|y_{n}-v\right\| \leq\left\|x_{n}-v\right\|\right\}, \\
x_{n+1}=P_{C_{n+1}} x_{1}, \quad n \geq 1,
\end{array}\right.
$$

where $\left\{u_{n, m}\right\}$ is such that

$$
\varphi_{m}\left(u_{m}\right)-\varphi_{m}\left(u_{n, m}\right)+\frac{1}{r_{n, m}}\left\langle u_{m}-u_{n, m}, u_{n, m}-x_{n}\right\rangle \geq 0, \quad \forall u_{m} \in C
$$

for each $1 \leq m \leq N$. Assume that the above sequence also satisfies the following restrictions:

(a) $\alpha_{n} \leq a<1$;

(b) $\sum_{m=1}^{N} \beta_{n, m}=1$ and $0 \leq b \leq \beta_{n, m}<1$ for each $1 \leq m \leq N$;

(c) $0<c \leq r_{n, m} \leq d<\infty$ for each $1 \leq m \leq N$,

where $a, b, c$ and $d$ are real numbers. Then the sequence $\left\{x_{n}\right\}$ strongly converges to $\operatorname{Proj}_{\mathcal{F}} x_{1}$.

Proof Putting $S=I, A_{m}=B=0$ and $F_{m}(x, y)=\varphi(y)-\varphi(x)$, we find from Theorem 3.1 the desired conclusion immediately.

\section{Competing interests}

The author declares that he has no competing interests.

\section{Acknowledgements}

The author is grateful to the reviewers for useful suggestions which improved the contents of the article.

Received: 7 November 2012 Accepted: 25 February 2013 Published: 18 March 2013

\section{References}

1. Lin, L, Tu, Cl: The studies of systems of variational inclusions problems and variational disclusions problems with applications. Nonlinear Anal. 69, 1981-1998 (2008)

2. Cho, SY, Kang, SM: Approximation of fixed points of pseudocontraction semigroups based on a viscosity iterative process. Appl. Math. Lett. 24, 224-228 (2011)

3. Yang, S, Li, W: Iterative solutions of a system of equilibrium problems in Hilbert spaces. Adv. Fixed Point Theory 1 , $15-26(2011)$

4. Blum, E, Oettli, W: From optimization and variational inequalities to equilibrium problems. Math. Stud. 63, 123-145 (1994)

5. Cho, SY, Kang, SM: Approximation of common solutions of variational inequalities via strict pseudocontractions. Acta Math. Sci. 32, 1607-1618 (2012)

6. Qin, X, Chang, SS, Cho, YJ: Iterative methods for generalized equilibrium problems and fixed point problems with applications. Nonlinear Anal. 11, 2963-2972 (2010)

7. Lv, S, Wu, C: Convergence of iterative algorithms for a generalized variational inequality and a nonexpansive mapping. Eng. Math. Lett. 1, 44-57 (2012)

8. Zegeye, $\mathrm{H}$, Shahzad, N: Strong convergence theorem for a common point of solution of variational inequality and fixed point problem. Adv. Fixed Point Theory 2, 374-397 (2012)

9. Combettes, PL, Hirstoaga, SA: Equilibrium programming in Hilbert spaces. J. Nonlinear Convex Anal. 6, 117-136 (2005)

10. Luo, $\mathrm{H}$, Wang, Y: Iterative approximation for the common solutions of a infinite variational inequality system for inverse-strongly accretive mappings. J. Math. Comput. Sci. 2, 1660-1670 (2012)

11. Qin, X, Cho, YJ, Kang, SM: Convergence theorems of common elements for equilibrium problems and fixed point problems in Banach spaces. J. Comput. Appl. Math. 225, 20-30 (2009)

12. Al-Bayati, AY, Al-Kawaz, RZ: A new hybrid WC-FR conjugate gradient-algorithm with modified secant condition for unconstrained optimization. J. Math. Comput. Sci. 2, 937-966 (2012)

13. Reddappa, B, Reddy, MVS, Prasad, KR: Effect of variable viscosity on the peristaltic flow of a Newtonian fluid in asymmetric channel under the effect of a magnetic field. J. Math. Comput. Sci. 2, 1598-1611 (2012)

14. Qin, X, Cho, SY, Kang, SM: On hybrid projection methods for asymptotically quasi- $\boldsymbol{\phi}$-nonexpansive mappings. Appl. Math. Comput. 215, 3874-3883 (2010) 
15. Khader, MM, Mohamed, ST: Numerical treatment for first order neutral delay differential equations using spline functions. Eng. Math. Lett. 1, 32-43 (2012)

16. Liu, Z, Agarwal, RP, Feng, C, Kang, SM: Weak and strong convergence theorems of common fixed points for a pair of nonexpansive and asymptotically nonexpansive mappings. Acta Univ. Palacki. Olomuc., Fac. Rerum Nat., Math. 44 83-96 (2005)

17. Qin, $\mathrm{X}, \mathrm{Cho}, \mathrm{YJ}$, Kang, SM, Zhou, H: Convergence of a modified Halpern-type iteration algorithm for quasi- $\boldsymbol{\phi}$-nonexpansive mappings. Appl. Math. Lett. 22, 1051-1055 (2009)

18. Mann, WR: Mean value methods in iteration. Proc. Am. Math. Soc. 4, 506-510 (1953)

19. Genel, A, Lindenstrass, J: An example concerning fixed points. Isr. J. Math. 22, 81-86 (1975)

20. Haugazeau, Y: Sur les inéquations variationnelles et la minimisation de fonctionnelles convexes. Ph.D. Thesis, Université de Paris (1968)

21. Ye, J, Huang, J: Strong convergence theorems for fixed point problems and generalized equilibrium problems of three relatively quasi-nonexpansive mappings in Banach spaces. J. Math. Comput. Sci. 1, 1-18 (2011)

22. Qin, X, Cho, SY, Kang, SM: Strong convergence of shrinking projection methods for quasi- $\boldsymbol{\phi}$-nonexpansive mappings and equilibrium problems. J. Comput. Appl. Math. 234, 750-760 (2010)

23. Kim, JK: Strong convergence theorems by hybrid projection methods for equilibrium problems and fixed point problems of the asymptotically quasi- $\phi$-nonexpansive mappings. Fixed Point Theory Appl. 2011, Article ID 10 (2011)

24. Kang, SM, Cho, SY, Liu, Z: Convergence of iterative sequences for generalized equilibrium problems involving inverse-strongly monotone mappings. J. Inequal. Appl. 2010, Article ID 827082 (2010)

25. Kim, JK: Hybrid projection algorithms for generalized equilibrium problems and strictly pseudocontractive mappings. J. Inequal. Appl. 2010, Article ID 312602 (2010)

26. Wu, C, Liu, A: Strong convergence of a hybrid projection iterative algorithm for common solutions of operator equations and of inclusion problems. Fixed Point Theory Appl. 2012, Article ID 90 (2012)

27. Qin, X, Cho, SY, Kang, SM: An extragradient-type method for generalized equilibrium problems involving strictly pseudocontractive mappings. J. Glob. Optim. 49, 679-693 (2011)

28. Lu, J, Gu, F: Projection methods of iterative solutions in Hilbert spaces. Fixed Point Theory Appl. 2012, Article ID 162 (2012)

29. Nilsrakoo, W, Saejung, S: Strong convergence theorems by Halpern-Mann iterations for relatively nonexpansive mappings in Banach spaces. Appl. Math. Comput. 217, 6577-6586 (2011)

30. Nilsrakoo, W, Saejung, S: Weak and strong convergence theorems for countable Lipschitzian mappings and its applications. Nonlinear Anal. 69, 2695-2708 (2008)

31. Nilsrakoo, W, Saejung, S: Strong convergence theorems for a countable family of quasi-Lipschitzian mappings and its applications. J. Math. Anal. Appl. 356, 154-167 (2009)

32. Qin, X, Shang, M, Su, Y: Strong convergence of a general iterative algorithm for equilibrium problems and variational inequality problems. Math. Comput. Model. 48, 1033-1046 (2008)

33. Takahashi, S, Takahashi, W: Viscosity approximation methods for equilibrium problems and fixed point problems in Hilbert spaces. J. Math. Anal. Appl. 331, 506-515 (2007)

34. Takahashi, S, Takahashi, W: Strong convergence theorem for a generalized equilibrium problem and a nonexpansive mapping in a Hilbert space. Nonlinear Anal. 69, 1025-1033 (2008)

35. Zhang, M: Iterative algorithms for common elements in fixed point sets and zero point sets with applications. Fixed Point Theory Appl. 2012, Article ID 21 (2012)

36. Qing, Y, Kim, JK: Weak convergence of algorithms for asymptotically strict pseudocontractions in the intermediate sense and equilibrium problems. Fixed Point Theory Appl. 2012, Article ID 132 (2012)

37. Rockafellar, RT: On the maximality of sums of nonlinear monotone operators. Trans. Am. Math. Soc. 149, 75-88 (1970)

doi:10.1186/1687-1812-2013-59

Cite this article as: Chen: A strong convergence theorem of common elements in Hilbert spaces. Fixed Point Theory and Applications 2013 2013:59.

\section{Submit your manuscript to a SpringerOpen ${ }^{\circ}$ journal and benefit from:}

- Convenient online submission

- Rigorous peer review

- Immediate publication on acceptance

- Open access: articles freely available online

- High visibility within the field

- Retaining the copyright to your article 